\title{
Apprendre dans les réorganisations : une perspective communicationnelle
}

Patrick Gilbert

\section{(2) OpenEdition}

12 Journals

Édition électronique

URL : http://journals.openedition.org/communicationorganisation/435

DOI : 10.4000/communicationorganisation.435

ISSN : $1775-3546$

Éditeur

Presses universitaires de Bordeaux

Édition imprimée

Date de publication : 1 juin 2008

Pagination : $40-48$

ISBN : 978-2-86781-506-5

ISSN : $1168-5549$

Référence électronique

Patrick Gilbert, «Apprendre dans les réorganisations : une perspective communicationnelle », Communication et organisation [En ligne], 33 | 2008, mis en ligne le 01 juin 2011, consulté le 02 mai 2019. URL : http://journals.openedition.org/communicationorganisation/435; DOI : 10.4000/ communicationorganisation.435 
Dossier : Conduire le changement organisationnel?

\title{
Résumé
}

Les réorganisations ponctuent désormais la vie des entreprises de toutes tailles et de tous secteurs. Il s'agit d'opérations souvent complexes et risquées. En cas d'échec, elles fragilisent l'entreprise et déstabilisent son personnel. Même en cas de succès, elles restent délicates. La qualité des apprentissages collectifs réalisés dans le cours même du changement conditionne le bon fonctionnement des organisations transformées. Or, la pratique n'accorde que peu d'attention à ce point; changement et apprentissage sont habituellement pensés dans des temporalités et dans des lieux distincts et par des acteurs différents. Deux études de cas sont proposées pour illustrer à la fois les apprentissages spontanés et les tentatives d'apprentissages délibérés mises en place par les entreprises.

\section{Mots-clés}

Apprentissage organisationnel, réorganisation, processus de changement, apprentissage spontané, apprentissage délibéré

\begin{abstract}
Reorganizations are now a routine part of the life of companies of all sizes and in all sectors. These reorganizations are often complex and risky. If the reorganizations fail, they weaken the company and destabilize its workforce. And even if they succeed, they may remain precarious. The quality of organizational learnings during the change process will affect how well the organization functions after transformation. Still, little attention is paid to this point in practice. Typically, the change process and organizational learnings are disconnected in terms of time, place and people. Two case studies are offered to illustrate both spontaneous adaptation to change and the more deliberate application of organization learnings in the change process.
\end{abstract}

\section{Key-Words}

Organizational learning, reorganization, change process, spontaneous learning, deliberate learning

\section{Patrick GILBERT}

Patrick Gilbert est professeur en sciences de gestion à l'IAE de Paris (Université Panthéon-Sorbonne). Il est aussi chercheur au GREGOR (Groupe de Recherche en Gestion des Organisations). 


\title{
Apprendre dans les réorganisations : une perspective communicationnelle
}

\author{
Patrick Gilbert
}

patrick.gilbert@univ-paris1.fr

Les réorganisations ponctuent désormais la vie des entreprises de toutes tailles et de tous secteurs. Leurs formes concrètes sont multiples: externalisation d'activités, fermeture ou déplacement physique de services, suppression d'échelons hiérarchiques, mise en place de structures matricielles, déploiement de systèmes de gestion intégrés... Il s'agit d'opérations complexes et risquées. Les écueils sont nombreux et leurs conséquences économiques peuvent être fatales. En cas d'échec, elles fragilisent l'entreprise et déstabilisent son personnel. En cas de succès, elles restent délicates. Bien que ce thème fasse l'objet d'une attention grandissante par le management, une question demeure peu traitée : celle des apprentissages nécessaires au fonctionnement de la nouvelle organisation et des interactions et «modalités de la communication» (Mucchielli, 1991) entre les acteurs de la réorganisation. Pour éclairer cette question, nous nous inscrirons dans le cadre d'une approche processuelle du changement organisationnel (Weick, 1979 ; Starbuck, 1983 ; Giroux et Dumas, 1997).

\section{Entre structure formelle et structure des échanges}

Depuis les travaux de l'école des relations humaines, l'analyse des organisations distingue deux structures en tension créatrice, l'une formelle et l'autre informelle.

La première perspective, celle de la structure formelle, correspond à l'approche classique, pour laquelle la structure est un instrument au service du groupe dirigeant. Elle porte sur le partage explicite des activités et des responsabilités et aboutit à la fixation d'attributs codifiés (organigramme, procédures, normes écrites de fonctionnement). Dans ce cas, «réorganisation» signifie essentiellement modification de la division du travail et déplacement des lieux d'exercice de l'autorité. 


\section{Dossier : Conduire le changement organisationnel?}

Dans la seconde perspective, la structure informelle est la résultante d'un ensemble d'interactions entre acteurs poursuivant leurs propres buts. Selon Contandriopoulos et al. (2005), cette structure, qualifiée de «structure des échanges», est la résultante d'un ensemble d'interactions entre acteurs qui définissent à la fois la densité des échanges, la nature de ce qui circule dans ces interactions et le mode d'interaction (plus ou moins conflictuel, plus ou moins coopératif). Ici parler de réorganisation c'est évoquer les changements introduits dans les relations entre acteurs.

L'opposition historique entre le formel et l'informel est bien sûr réductrice. Les deux structures sont inséparables et inextricables. Pour nous, leur convergence dépend d'une autre réorganisation, moins évidente, celle des apprentissages. Dans cette perspective une réorganisation est un processus d'apprentissage collectif dans lequel se confrontent deux régulations (Reynaud, 1988): la régulation de contrôle, qui détermine l'énoncé et l'exécution des nouvelles règles «officielles » (le formel) et la régulation autonome, qui cherche à faire valoir des pratiques informelles. C'est dans l'acceptation d'une confrontation entre ces deux régulations qu'émergeront les nouveaux fonctionnements. Cette perspective de type communicationnel s'intéresse à la manière dont la connaissance est produite dans l'action et se fonde sur le débat, la gestion de la mise en commun, la capitalisation des expériences.

Dans une réorganisation, il y a toujours a minima un apprentissage spontané ou «naturel » qui emprunte au registre de l'informel. Par essais, erreurs et réajustements, les individus tentent de faire face aux situations nouvelles rencontrées. Ils y parviennent tant bien que mal. Cette situation peut être voulue au nom de l'autonomie individuelle : à l'acteur de «s'arranger » avec l'incertitude.

Cependant, la variété des réponses face à une même situation-problème rend difficile le travail collectif. Aussi, certaines entreprises tentent bien d'organiser les apprentissages. Mais leur réponse la plus habituelle relève d'une planification des acquisitions $a$ priori. Par exemple, on s'appuiera sur des référentiels de compétences ${ }^{1}$, pouvant découler eux-mêmes d'une analyse du travail prescrit et, en amont, d'organisations-cibles. Dans le prolongement de cette analyse, on élaborera des stages de formation destinés à préparer

\footnotetext{
1 «Vivre et agir dans l'incertitude» pourra d'ailleurs compter parmi les «compétences » formulées.
} 
aux nouveaux rôles professionnels. On reste bien ainsi dans le registre d'une régulation de contrôle.

Pour progresser dans la compréhension du rôle des apprentissages collectifs dans les réorganisations, nous avons privilégié deux terrains d'enquête pour lesquels nous avons un recul ${ }^{2}$ d'une dizaine années. Au-delà des différences (secteur, activités, populations concernées) qui, superficiellement, opposent ces cas, les difficultés d'apprentissage qu'on y découvre sont, nous allons le voir, très similaires.

\section{Cas $n^{\circ} 1$ : l'organisation lacunaire des stations du métro parisien}

La situation qui prévalait dans les stations du métro parisien jusqu'à la fin des années 1980 était celle d'un système de travail fortement hiérarchisé et structuré par une abondante réglementation et des procédures laissant peu de place à l'initiative. Les employés, agents de station de qualification unique, sont affectés dans les différents postes selon une logique habituelle dans les grandes bureaucraties : les agents les plus récents et les moins expérimentés occupent les postes les moins prisés. Les stations sont exploitées selon leur taille avec un ou plusieurs agents selon des modalités d'organisation du travail uniforme.

La modernisation, lancée au début des années 1990, s'inscrit dans une mise en place de la décentralisation et des inflexions managériales qui l'accompagnent: autonomie reconnue aux lignes en tant qu'unités opérationnelles, mise en place de métiers de développement, rôle confié à la maîtrise en matière d'évaluation du personnel. L'entreprise, dans laquelle primait jusqu'à présent une logique de gestion technique et administrative de l'offre de transport collectif, s'est engagée, dans une stratégie d' «orientation client» visant à adapter l'offre aux besoins et aux attentes de ses usagers.

De nouvelles transformations sont engagées afin d'améliorer la qualité $\mathrm{du}$ service offert aux voyageurs. Pour atteindre cet objectif, la direction de l'entreprise mise à la fois sur le développement et la modernisation des moyens techniques et sur la mise en place d'une organisation dont la souplesse permettrait de diversifier l'offre de service. La réorganisation s'effectue à effectif constant.

${ }^{2}$ Ce recul nous permet de citer nommément les entreprises concernées, les réorganisations évoquées et les problèmes que leur mise en œuvre soulevait appartenant désormais à l'histoire. 


\section{Dossier : Conduire le changement organisationnel?}

Les responsables des lignes seront décentralisés en partie sur des secteurs regroupant un ensemble de stations sous l'autorité d'un cadre et d'agents de maîtrise polyvalents. Chaque station sera équipée d'interphones, de caméras, de télé-afficheurs et de distributeurs automatiques de titres de transport. Des agents de station, ainsi «libérés» de la vente, pourront participer à des équipes mobiles patrouillant sur un secteur. Des centres de liaison seront créés avec des moniteurs de télésurveillance et des équipements de communication (liaisons radio avec des équipes mobiles, avertisseurs d'alarme, etc). Compte tenu de l'ampleur du changement envisagé, du coût des investissements et de la rupture culturelle qu'il représente, le parti est pris d'expérimenter le projet sur deux lignes, considérées comme représentatives du réseau.

Dans cette phase expérimentale, les fonctions ne sont volontairement que peu précisées. Si les objectifs particuliers et les objectifs globaux sont fixés, les tâches et les procédures d'application ne le sont que partiellement. Chacun des responsables a la charge de définir au fur et à mesure les pratiques professionnelles les mieux adaptées. La vente de billets, autrefois centrale, n'est plus que l'un des rôles à tenir. L'agent de station intervient désormais en milieu ouvert, en relation avec les agents de conduite et les clients, confronté à des situations comportant une part d'inattendu, il doit s'adapter à l'événement.

Les vides dans la définition de l'organisation en creux étaient censés être remplis par les initiatives des agents. Or ce que l'on constate est souvent tout différent. Les agents privilégient les comportements qu'ils connaissent et, sauf directives contraires, retournent aux pratiques ou situations professionnelles antérieures. Par exemple, le travail à deux au guichet de vente, forme de travail bien éprouvée, tend à se reconstituer : tout naturellement un agent d'équipe mobile rejoint l'animateur de station qui lui en fait la demande pour reconstituer le couple «chef de station-receveur » de l'ancien modèle d'organisation.

\section{Cas $n^{\circ} 2$ : L'organisation éclatée d'établissements bancaires régionaux}

Depuis 1988, et surtout dans le courant des années 1990, le Crédit Agricole a engagé des fusions d'établissements implantés localement, afin d'accroître ses potentialités commerciales et financières. De 1988 à 2005, le nombre des caisses régionales a été ramené de 94 à 41, par vagues de fusion regroupant 2,3 , voire 4 caisses. Ces changements renvoient aux raisons fondamentales qui président aux décisions de 
fusion dans les réseaux bancaires (constituer des banques régionales possédant une taille critique en rapport avec les potentialités de leur région, réaliser des économies d'échelle à la fois sur le plan commercial, en renforçant l'efficacité commerciale et sur le plan de la productivité du travail).

Au-delà des grands choix stratégiques, fixés dans un cadre général par la Fédération Nationale du Crédit Agricole, qui vont définir la nouvelle banque régionale, la façon de conduire l'ensemble des opérations de rapprochement peut différer sensiblement d'une fusion à l'autre.

Deux banques régionales, plus particulièrement étudiées, avaient été constituées, au stade où nous les avons observées, par regroupement de trois établissements d'implantations départementales. Les sièges administratifs avaient été conservés avec une redistribution des activités entre implantations. Nous nous limiterons ici à évoquer la transformation des rôles des dirigeants et les compétences que ces transformations mobilisaient.

Lors d'une fusion, la proximité relationnelle entre l'encadrement, les services fonctionnels et les équipes à la base se réduit, en raison de l'effet de taille et de la mobilité. Cela implique de construire de nouveaux modes de régulation, tant en ce qui concerne la conduite des équipes que la gestion des personnes. La situation où un cadre dirigeant (chef de division ou de département) est appelé à coordonner les activités de collaborateurs se trouvant dispersés sur trois sites est habituelle. Les réponses constatées à cette situation sont diverses :

- à l'image de son directeur général, ce cadre dispose d'un bureau sur chacun des sites ;

- chaque vendredi, les collaborateurs directs de celui-ci viennent à leur tour faire le point de la situation et recevoir leurs consignes ;

- cet autre cadre fait sa tournée des trois établissements administratifs, soit de façon systématique, soit sur la base de priorités conjoncturelles ;

- un autre encore redéfinit en concertation avec son équipe ses modes de supervision et de contrôle de façon à limiter les déplacements physiques entre entités.

Les trois premières réponses sont de simples reproductions de comportements acquis : chacun à sa manière s'efforce de maintenir la relation de proximité physique avec ses collaborateurs et de préserver 
Dossier : Conduire le changement organisationnel?

la supervision directe qui prévalait dans l'organisation antérieure. Seule, la réponse « $d$. » témoigne d'un apprentissage collectif.

\section{L'organisation des apprentissages dans les deux cas}

Les deux cas montrent bien les limites d'un apprentissage abandonné à l'initiative individuelle. Les individus n'inventent pas spontanément des modes d'action adaptés aux nouvelles organisations - pas plus que ces dernières n'engendrent automatiquement les comportements qui leur seraient nécessaires pour fonctionner. La tendance dominante de chacun est de s'inspirer directement des conduites professionnelles qui ont fait leur preuve dans le passé, de tenter de réactiver face aux situations risquées les anciennes manières de faire (Weick, 1990), les routines défensives (Argyris, 1985).

Les deux entreprises en étaient conscientes. Il existait, dans les deux cas, des dispositifs orientés vers l'apprentissage des salariés. Le département Métro de la RATP voulait tirer, après six mois de fonctionnement, les enseignements utiles d'une phase d'expérimentation, avant de décider d'étendre son nouveau modèle d'organisation. La Fédération du Crédit Agricole, aux deux tiers d'un parcours de fusions à grande échelle, dont chacune a fait l'objet d'une préparation minutieuse, s'interrogeait sur le parti à tirer d'une expérience de plusieurs années.

La construction de référentiels de compétences a été engagée dans les deux cas. Construire des référentiels collectifs sur le travail ne manque pas d'intérêt et il existe désormais sur le sujet un savoir-faire établi et des réflexions de fond. Mais la dimension prospective de tels référentiels, ici essentielle, est problématique. En effet, comment formuler ce que les salariés devront mobiliser dans une situation future dont les contours resteront flous, jusqu'à ce que les activités soient effectivement exercées?

La formation aux nouveaux emplois fait également partie de la batterie de moyens auxquels il est facile de penser. Ce moyen a été mobilisé à la RATP, comme au Crédit Agricole. Mais là encore, on est limité par la méconnaissance d'un travail dont le mode de réalisation est, pour une large part, en construction. On est donc tenté de se centrer sur les seuls aspects techniques du changement organisationnel (les nouvelles procédures, les nouveaux outils).

Dans le cas de l'entreprise de transport, dans l'une des deux lignes expérimentales, un groupe de suivi avait été mis en place. Les participants du groupe questionnaient l'organisation du travail, les 
règles et les procédures, les dispositifs techniques en place ainsi que les choix de management, en relation avec les difficultés rencontrées pour faire fonctionner la nouvelle organisation. En raison du caractère un peu dérangeant des débats dans lesquels les questions exprimées n'appelaient pas de réponses simples, la direction de la ligne avait, au bout de quelques semaines, décidé d'y mettre fin. Cet incident montre bien qu'une réorganisation implique des modifications des relations de travail et de pouvoir, de nouvelles articulations entre formel et informel - ce que Reynaud (1988) appelle une «régulation conjointe » - et cela n'est pas un changement anodin.

Dans les banques régionales, nous avons été invité à aider les acteurs des fusions à réfléchir au pourquoi du succès, pour permettre un partage d'expérience profitable à l'ensemble de l'entreprise. Le dispositif d'évaluation constituait également un dispositif d'apprentissage dialogique, fondé sur la narration (Giroux et Marroquin, 2005), allant du simple témoignage à la confrontation des expériences dans des groupes d'analyse des pratiques, entre les acteurs des réorganisations, puis avec les instances de direction générale. La confrontation est reconnue comme la plus susceptible d'entraîner un apprentissage profond et durable (Bouwen et Fry, 1993). Mais cette modalité interactionnelle ne va pas de soi.

\section{Une perspective qui ne va pas de soi}

Dans les réorganisations, les individus et les groupes doivent, d'une part, désapprendre certaines pratiques issues des manières de faire qui prévalaient dans l'ancienne organisation et, d'autre part, se constituer de nouvelles représentations collectives sur la façon de travailler ensemble. Ces dernières ne peuvent être entièrement inculquées a priori, dans des apprentissages cadrés, conçus indépendamment d'une connaissance vécue des situations de travail. Mais l'intelligence de l'expérimentation qui implique une réflexion dans l'action et un débat rencontre divers obstacles.

Tout d'abord, il est exigeant et contraignant pour une entreprise de mettre en place des dispositifs expérimentaux qui supposent l'acceptation de l'incertitude ainsi que des jeux politiques qu'elle entraîne. On comprend la préférence habituelle pour les modus operandi linéaires dont le design est plus rassurant. A l'inverse, comme l'a montré le cas de la RATP, un design incomplet ne favorise pas toujours la créativité.

Un autre obstacle, de type dialogique, tient à l'explicitation et au partage d'expériences. Toute expérience se caractérise par une 
Dossier : Conduire le changement organisationnel?

difficulté intrinsèque, qui est celle de sa transmission aux autres. L'appel à la spontanéité des acteurs est rarement suffisant, Il peut être utile de stimuler les confrontations pour favoriser ce transfert.

\section{Bibliographie}

ARGYRIS, Chris. Strategy, Change and Defensive Routines. Boston : MA, Ballinger, 1985, 368 p.

BOUWEN, Rene, FRY, Ronald. Innovation et apprentissage organisationnel. Communication et Organisation, n³, mai 1993, p. $129-157$.

CONTANDRIOPOULOS, Damien, CONTANDRIOPOULOS, André-Pierre, DENIS, Jean-Louis, VALETTE, Annick. L'hôpital en restructuration. Regards croisés sur la France et le Québec. Montréal : Presses Universitaires de Montréal, 2005, 302 p.

GIROUX, Nicole, DUMAS, Dany. Trois modalités d'intégration des fusions-acquisitions: l'intégration planifiée, l'intégration émergente et l'intégration conjointe. Actes de la conférence de l'AIMS, HÉC, Montréal, 1997.

GIROUX, Nicole, MARROQUIN, Lissette. L'approche narrative des organisations. Revue française de gestion, $\mathrm{n}^{\circ} 159$, nov-déc., 2005, p. $15-45$.

MUCCHIELLI, Alex. Les situations de communication. Paris : Eyrolles, 1991, $131 \mathrm{p}$.

REYNAUD, Jean-Daniel. Les régulations dans les organisations: régulation de contrôle et régulation autonome. Revue Française de Sociologie, XXIX, 1988, p. 5-18.

STARBUCK, William H. Organizations as Action Generators. American Sociological Review, n48, 1983, p. 91-102.

WEICK, Karl. The Social Psychology of Organizing. Readings, Mass : Addison Westley, 2e éd., 1979 [1969]), 294 p.

WEICK, Karl. The vulnerable system: An analysis of the Tenerife air disaster. Journal of Management, 1990, vol.16, n³ 3, p. 571-593. 
